PROCEEDINGS OF THE

AMERICAN MATHEMATICAL SOCIETY

Volume 130, Number 11, Pages 3165-3170

S 0002-9939(02)06422-5

Article electronically published on March 25, 2002

\title{
NATURAL BOUND IN KWIECIŃSKI'S CRITERION FOR FLATNESS
}

\author{
JANUSZ ADAMUS
}

(Communicated by Wolmer V. Vasconcelos)

\begin{abstract}
Kwieciński has proved a geometric criterion for flatness: A morphism $f: X \rightarrow Y$ of germs of analytic spaces is not flat if and only if its $i$-fold fibre power $f^{\{i\}}: X^{\{i\}} \rightarrow Y$ has a vertical component, for some $i$. We show how to bound $i$ using Hironaka's local flattener: If $f$ is not flat, then $f^{\{d\}}$ has a vertical component, where $d$ is the minimal number of generators of the ideal in $\mathcal{O}_{Y}$ of the flattener of $X$.
\end{abstract}

\section{INTRODUCTION}

Let $f: Z \rightarrow Y$ be a morphism of germs of analytic spaces, and let $W$ be an irreducible component of $Z$. Let $\mathcal{P}$ be the associated prime of the zero ideal in the local ring $\mathcal{O}_{Z}$, corresponding to $W$. We say that the component $W$ is vertical if there exists a nonzero $a \in \mathcal{O}_{Y}$ with $f^{*} a \in \mathcal{P}$ (see also the remark following Lemma 3.1).

In [11, Thm. 1.1], Kwieciński shows that assuming $Y$ is irreducible, i.e. the local ring $\mathcal{O}_{Y}$ is a domain, the following conditions are equivalent:

(i) $f: X \rightarrow Y$ is flat;

(ii) for any $i \geq 1$, the canonical map $\underbrace{X \times_{Y} \ldots \times_{Y} X}_{i \text { times }} \rightarrow Y$ has no (isolated or embedded) vertical components.

From now on we will write $X^{\{i\}}$ for the space $X \times_{Y} \ldots \times_{Y} X$ (i times) and $f^{\{i\}}$ for the canonical map $X^{\{i\}} \rightarrow Y$.

Note that if $f$ is flat, then $f^{\{i\}}$ is flat for all $i$, since flatness is preserved by any base change ([9, §6, Prop. 8]) and the composition of flat maps is flat. Therefore the implication (i) $\Rightarrow$ (ii) is an immediate consequence of the definition of flatness in terms of relations (see e.g. [2, Prop. 7.3]). This in fact is the only place where the irreducibility assumption is needed (cf. the example in this section below).

Implication (ii) $\Rightarrow$ (i) does not require irreducibility of $Y$, by Kwieciński's Lemma 3.1 below. Thus, for any nonflat map $f: X \rightarrow Y$ of germs of analytic spaces there is a positive integer $i$ such that $X^{\{i\}}$ has a vertical component. The proof given by Kwieciński is based on Hironaka's criterion for flatness (Thm. 2.2 below, see also [2] Thm. 7.9]). Hironaka uses this criterion to prove the existence of the local flattener (see [2, Thm. 7.12]), which we use to give a precise power

Received by the editors March 19, 2001 and, in revised form, June 11, 2001.

2000 Mathematics Subject Classification. Primary 14B25, 13C11.

Key words and phrases. Fibre product, vertical component, local flattener. 
needed in condition (ii) of Kwieciński's theorem. The local flattener for a morphism $f: X \rightarrow Y$ of germs of analytic spaces is, by definition, the maximal subgerm $P$ of $Y$ such that $\mathcal{O}_{X} \otimes_{\mathcal{O}_{Y}} \mathcal{O}_{P}$ is $\mathcal{O}_{P}$-flat, i.e. $f \mid f^{-1}(P): f^{-1}(P) \rightarrow P$ is a flat morphism (cf. [2, Thm. 7.12]). Our main result is the following:

Theorem 1.1. Let $f: X \rightarrow Y$ be a nonflat morphism of germs of analytic spaces. Let $Q$ be the ideal in $\mathcal{O}_{Y}$ of the flattener of $X$, and let $d$ be the minimal number of generators of $Q$. Then the canonical map $f^{\{d\}}: X^{\{d\}} \rightarrow Y$ has a vertical component.

Of course, this leaves open the question of calculation. First results have been given by Vasconcelos [15], and Galligo and Kwieciński [6] under certain restrictions on $X$ and $Y$ : Galligo and Kwieciński assert that, assuming $X, Y$ are reduced, $X$ is of pure dimension, and $Y$ is smooth of dimension $n, f: X \rightarrow Y$ is flat if and only if the canonical map $f^{\{n\}}: X^{\{n\}} \rightarrow Y$ has no geometric vertical components (6. Thm. 6.1]). An analogous result in the algebraic category, but without the pure dimension assumption on $X$, was obtained by Vasconcelos in the case $\operatorname{dim} Y=2$ (15, Prop. 6.1]).

By a geometric vertical component of a morphism $f: Z \rightarrow Y$ we mean a component $W$ of $Z$ such that, for arbitrarily small representatives $\widetilde{W}, \widetilde{Y}, \tilde{f}$ of $W, Y, f$, respectively, the image $\tilde{f}(\widetilde{W})$ has empty interior in $\widetilde{Y}$ with transcendental topology (cf. [6] and 11]). Note that although the notions of vertical and geometric vertical coincide in the algebraic case over irreducible germ $Y$ (as the image of an algebraic set under a polynomial morphism is always constructible), they are not the same in the analytic setup.

Clearly, over irreducible $Y$, every vertical component is geometric vertical, but the converse is false in general. Consider for instance the Osgood mapping $f$ : $\mathbb{C}_{0}^{2} \rightarrow \mathbb{C}_{0}^{3}$ defined as $(x, y) \mapsto\left(x, x y, x y e^{y}\right)$ (see e.g. [7]). Then for an arbitrary neighbourhood $U$ of the origin in $\mathbb{C}^{2}, f(U)$ has empty interior in $\mathbb{C}^{3}$, but there is no proper analytic subgerm of $\mathbb{C}_{0}^{3}$ containing $(f(U))_{0}$, and hence $f$ has no vertical components in our sense.

Observe that in general, i.e. without the irreducibility assumption on $Y$, the equivalence from Kwieciński's theorem is no longer valid. Consider for instance the identity mapping on the space $X=\left\{(x, y) \in \mathbb{C}^{2}: x y=0\right\}$, which is obviously flat while each of the irreducible components of $X$ is vertical.

\section{Diagram OF INITIAL EXPONENTS AND HIRONAKA'S CRITERION FOR FLATNESS}

We briefly recall here basic facts regarding the diagram of initial exponents. For details we refer to [2].

Let $A$ be a local analytic $\mathbb{C}$-algebra, say $A=\mathbb{C}\left\{y_{1}, \ldots, y_{m}\right\} / J$, with the maximal ideal $\mathfrak{m}$. Let $L$ be a total ordering of monomials in $t=\left(t_{1}, \ldots, t_{n}\right)$ with coefficients in $A$ which is compatible with addition of exponents. We write $t^{\beta}$ for $t_{1}^{\beta_{1}} \ldots t_{n}^{\beta_{n}}$, where $\beta=\left(\beta_{1}, \ldots, \beta_{n}\right) \in \mathbb{N}^{n}$. Let $A\{t\}=\mathbb{C}\{y, t\} / J \cdot \mathbb{C}\{y, t\}$ be the ring of convergent power series in $t$ with coefficients in $A$. For a series $F=\sum_{\beta \in \mathbb{N}^{n}} a_{\beta} t^{\beta} \in A\{t\}$ define its evaluation at 0 as $F(0)=\sum_{\beta \in \mathbb{N}^{n}} a_{\beta}(0) t^{\beta} \in A / \mathfrak{m}\{t\}=\mathbb{C}\{t\}$, and for an ideal $I$ in $A\{t\}$ define $I(0)=\{F(0): F \in I\}$, the evaluated ideal. The support of $F$ is defined 
as supp $F=\left\{\beta \in \mathbb{N}^{n}: a_{\beta} \neq 0\right\}$, and $\nu_{L}(F)=\min _{L}\{\beta \in \operatorname{supp} F\}$ denotes the initial exponent of $F$ (with respect to $L$ ). Similarly, $\operatorname{supp} F(0)=\left\{\beta \in \mathbb{N}^{n}: a_{\beta}(0) \neq 0\right\}$ and $\nu_{L}(F(0))=\min _{L}\{\beta \in \operatorname{supp} F(0)\}$, for the evaluated series.

Let $I$ be an ideal in $A\{t\}$. The diagram of initial exponents of $I$ (with resp. to $L)$ is defined as $N_{L}(I)=\left\{\nu_{L}(F): F \in I\right\} \subset \mathbb{N}^{n}$.

Let $f: X \rightarrow Y$ be a morphism of germs of analytic spaces. Without loss of generality can assume that $X$ is a subgerm of $\mathbb{C}_{0}^{n}$, for some $n \geq 1$. We can then embed $X$ into $Y \times \mathbb{C}_{0}^{n}$ via the graph of $f$. Therefore the local ring $\mathcal{O}_{X}$ of $X$ can be thought of as a quotient of the local ring of $Y \times \mathbb{C}_{0}^{n}$, i.e. $\mathcal{O}_{X}=\mathcal{O}_{Y}\{t\} / I$, for some ideal $I$ in $\mathcal{O}_{Y}\{t\}$, where $t=\left(t_{1}, \ldots, t_{n}\right)$. Let $\Delta=\mathbb{N}^{n} \backslash N_{L}(I(0))$ be the complement of the diagram of initial exponents of the evaluated ideal $I(0)$, and define $\mathcal{O}_{Y}\{t\}^{\Delta}=\left\{F \in \mathcal{O}_{Y}\{t\}: \operatorname{supp} F \subset \Delta\right\}$. Now consider the canonical projection $\mathcal{O}_{Y}\{t\} \rightarrow \mathcal{O}_{X}$ and its restriction to $\mathcal{O}_{Y}\{t\}^{\Delta}$, called $\kappa$. The two results below, due to Hironaka, are crucial for our considerations.

Proposition 2.1 ([9 §6, Prop.9]). The natural map $\kappa: \mathcal{O}_{Y}\{t\}^{\Delta} \rightarrow \mathcal{O}_{X}=\mathcal{O}_{Y}\{t\} / I$ is surjective.

Theorem 2.2 ([9, $\S 6$, Prop.10]). With the notations above, the map $f: X \rightarrow Y$ is flat if and only if $\kappa$ is bijective.

Observe that $\operatorname{ker} \kappa=\left\{F \in \mathcal{O}_{Y}\{t\}^{\Delta}: F \in I\right\}$, i.e. ker $\kappa$ consists of these elements of the ideal $I$ whose supports lie entirely in $\Delta$.

Remark 2.3. Let $Q$ be the ideal in $\mathcal{O}_{Y}$ of the flattener of $X$. Then by the proof of [2] Thm. 7.12], $Q$ is generated by all the coefficients $a_{\beta}$ of all the series $F=\sum_{\beta \in \Delta} a_{\beta} t^{\beta}$ from $\operatorname{ker} \kappa$.

\section{KWIECIŃSKI'S LEMMA}

Our proof of Theorem 1.1 is based on the following result due to Kwieciński ([11. Lemma 3.2]).

Lemma 3.1. Let $f: X \rightarrow Y$ be a nonflat morphism of germs of analytic spaces. Then there is a positive integer $i$ such that there exists a nonzero $b \in \mathcal{O}_{X}{ }^{\{i\}}$ and a nonzero $a \in \mathcal{O}_{Y}$, with $a b=0$.

Observe that according to our definition, the condition above is equivalent to $X^{\{i\}}$ having a vertical component. Indeed, for $a \in \mathcal{O}_{Y}$ is a zerodivisor in $\mathcal{O}_{X^{\{i\}}}$ iff it belongs to some of the associated primes of the zero ideal in the local ring $\mathcal{O}_{X\{i\}}$.

Note also that without any assumptions on $Y$, flatness implies the following condition: For any $i \geq 1$, if $a \in \mathcal{O}_{Y}$ is a zerodivisor in $\mathcal{O}_{X}{ }^{\{i\}}$, then it is a zerodivisor in $\mathcal{O}_{Y}$. (By the definition of flatness in terms of relations.)

We will now sketch the main steps of the proof of Lemma 3.1: Since $f$ is not flat, Theorem 2.2 together with Proposition 2.1 imply that ker $\kappa \neq\{0\}$. Pick any nontrivial $F=\sum_{\beta \in \Delta} a_{\beta} t^{\beta}$ from ker $\kappa$. Let $a_{\beta_{1}}, \ldots, a_{\beta_{i}}$ be distinct nonzero coefficients of $F$ which generate the ideal in $\mathcal{O}_{Y}$ of all the coefficients of the series $F$. One then shows that $F=a_{\beta_{1}} F_{1}+\cdots+a_{\beta_{i}} F_{i}$, where $F_{j}(t)=t^{\beta_{j}}+\sum_{\beta \in \Delta \backslash\left\{\beta_{1}, \ldots, \beta_{i}\right\}} f_{\beta} t^{\beta} \in \mathcal{O}_{Y}\{t\}$, $j=1, \ldots, i$. Define $a_{j}=a_{\beta_{j}}$ and $h_{j}=\kappa\left(F_{j}\right)$ for $j=1, \ldots, i$. It follows that $a_{1} h_{1}+\cdots+a_{i} h_{i}=0$, but $h_{1}(0), \ldots, h_{i}(0)$ are linearly independent (over $\mathbb{C}$ ) in $\mathcal{O}_{X} / \mathfrak{m} \mathcal{O}_{X}=\mathbb{C}\{t\} / I(0)$, where $\mathfrak{m}$ is the maximal ideal in $\mathcal{O}_{Y}$. 
Next consider the following commutative diagram of canonical maps of $\mathcal{O}_{Y}$ modules, where both tensor products are taken $i$ times and we assume that $Y$ is a germ at 0 :

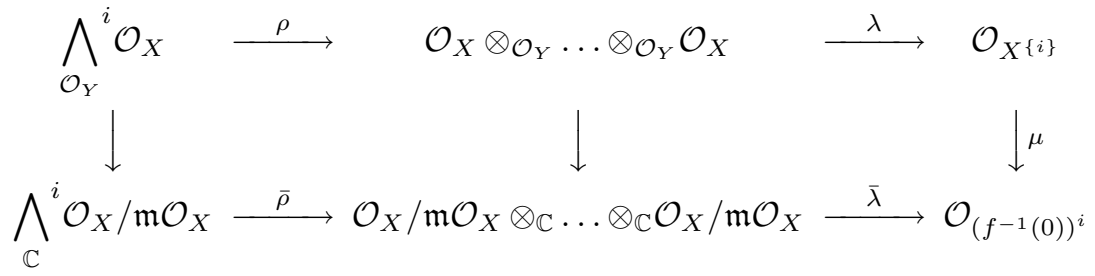

Put $a=a_{1}$ and $b=\lambda \circ \rho\left(h_{1} \wedge \cdots \wedge h_{i}\right)$. Since $a_{1} h_{1}$ is an $\mathcal{O}_{Y}$-linear combination of $h_{2}, \ldots, h_{i}$, then $a b=\lambda \circ \rho\left(\left(a_{1} h_{1}\right) \wedge h_{2} \cdots \wedge h_{i}\right)=0$. Finally $b \neq 0$, because $\mu(b)=\bar{\lambda} \circ \bar{\rho}\left(h_{1}(0) \wedge \cdots \wedge h_{i}(0)\right)$ is nonzero, as $h_{1}(0), \ldots, h_{i}(0)$ are linearly independent and $\bar{\rho}, \bar{\lambda}$ are injective.

\section{Proof of Theorem 1.1}

Let $d$ be the minimal number of generators of the flattener ideal $Q$, i.e. $d=$ $\operatorname{dim}_{\mathbb{C}} Q / \mathfrak{m} Q$, where $\mathfrak{m}$ is the maximal ideal in the local ring $\mathcal{O}_{Y}$. We begin with the following lemma.

Lemma 4.1. Assume $N_{L}(I(0))=\mathbb{N} \times D$ for some $D \subset \mathbb{N}^{n-1}$. Then there is a series $F \in \operatorname{ker} \kappa$ such that the coefficients of $F$ generate the ideal $Q$.

Remark 4.2. The condition $N_{L}(I(0))=\mathbb{N} \times D$ means that the diagram $N_{L}(I(0))$ is trivial in the $\beta_{1}$ direction. As can readily be seen from the proof below, we do not really need that much but only triviality in some of the $\beta_{1}, \ldots, \beta_{n}$ directions. The point is that triviality in the $\beta_{j}$ direction implies that for a series $F \in \mathcal{O}_{Y}\{t\}$ with $\operatorname{supp} F \subset \Delta$ and for any power $k$, the series $t_{j}^{k} F$ has support contained in $\Delta$ again, since $\operatorname{supp}\left(t_{j}^{k} F\right)=\operatorname{supp} F+(0, \ldots, k, \ldots, 0)$, with $k$ in the $j$ 'th place.

Proof of Lemma 4.1. Suppose to the contrary that for any series $F=\sum a_{\beta} t^{\beta}$ from ker $\kappa$, the coefficients $a_{\beta}$ of $F$ do not generate $Q$. Note that by Remark 2.3, all the coefficients of $F$ belong to $Q$.

For a series $F \in \operatorname{ker} \kappa$ define $d(F)$ as the maximal number of its coefficients linearly independent (over $\mathbb{C}=\mathcal{O}_{Y} / \mathfrak{m} \mathcal{O}_{Y}$ ) modulo $\mathfrak{m} Q$. It follows that for any $F$, $d(F)<d$, because otherwise the coefficients of some series would generate $Q$, by Nakayama's Lemma. Let $s=\max \{d(F) \mid F \in \operatorname{ker} \kappa\}$. Pick any $F_{1}=\sum_{\beta \in \Delta} a_{\beta} t^{\beta}$ from ker $\kappa$ with $d\left(F_{1}\right)=s$, and let $a_{\beta_{1}}, \ldots, a_{\beta_{s}}$ be its coefficients linearly independent modulo $\mathfrak{m} Q$. Then by the definition of $d\left(F_{1}\right)$, for any $\beta \in \operatorname{supp} F_{1} \backslash\left\{\beta_{1}, \ldots, \beta_{s}\right\}$ there exist $r_{\beta}^{1}, \ldots, r_{\beta}^{s} \in \mathbb{C}$ and $q_{\beta} \in \mathfrak{m} Q$ such that $a_{\beta}=r_{\beta}^{1} a_{\beta_{1}}+\cdots+r_{\beta}^{s} a_{\beta_{s}}+q_{\beta}$, i.e. all the other coefficients of $F$ are $\mathbb{C}$-linear combinations of $a_{\beta_{1}}, \ldots, a_{\beta_{s}}$ modulo $\mathfrak{m} Q$.

Since $s<d$, Remark 2.3, together with Nakayama's Lemma, implies that there exists a series $F_{2}=\sum_{\gamma \in \Delta} b_{\gamma} t^{\gamma} \in \operatorname{ker} \kappa$ such that for some $\gamma_{0} \in \operatorname{supp} F_{2}$, the coefficient $b_{\gamma_{0}}$ of $F_{2}$ is linearly independent from $a_{\beta_{1}}, \ldots, a_{\beta_{s}}$ modulo $\mathfrak{m} Q$. Fix such a series $F_{2}$ and take a positive integer $k$ satisfying inequality

$$
(k, 0, \ldots, 0)>\max \left\{\beta_{1}, \ldots, \beta_{s}\right\}
$$

with respect to the total ordering (induced by) $L$ in $\mathbb{N}^{n}$. 
Define a new series $F_{0}=F_{1}+t_{1}^{k} F_{2}$ and observe that $F_{0} \in$ ker $\kappa$. Indeed, $F_{2} \in \operatorname{ker} \kappa$ if and only if $F_{2} \in I$ and $\operatorname{supp} F_{2} \subset \Delta$. Therefore Remark 4.2 yields $\operatorname{supp}\left(t_{1}^{k} F_{2}\right) \subset \Delta$ (and obviously $t_{1}^{k} F_{2} \in I$ ), whence $t_{1}^{k} F_{2} \in \operatorname{ker} \kappa$ and $F_{0} \in \operatorname{ker} \kappa$.

Put $F_{0}=\sum_{\beta \in \Delta} c_{\beta} t^{\beta}$. By the inequality $(*), \operatorname{supp}\left(t_{1}^{k} F_{2}\right)>\max \left\{\beta_{1}, \ldots, \beta_{s}\right\}$, so in particular $c_{\beta_{1}}=a_{\beta_{1}}, \ldots, c_{\beta_{s}}=a_{\beta_{s}}$. Moreover, if $\beta_{0}=\gamma_{0}+(k, 0, \ldots, 0)$, then $\beta_{0} \neq \beta_{j}, j=1, \ldots, s$, and

$$
c_{\beta_{0}}=r_{\beta_{0}}^{1} a_{\beta_{1}}+\cdots+r_{\beta_{0}}^{s} a_{\beta_{s}}+q_{\beta_{0}}+b_{\gamma_{0}},
$$

where $r_{\beta_{0}}^{j} \in \mathbb{C}, j=1, \ldots, s, q_{\beta_{0}} \in \mathfrak{m} Q$. But $b_{\gamma_{0}}$ is linearly independent from $a_{\beta_{1}}, \ldots, a_{\beta_{s}}$, which implies that $c_{\beta_{0}}$ is linearly independent from $c_{\beta_{1}}, \ldots, c_{\beta_{s}}$. Thus $c_{\beta_{0}}, c_{\beta_{1}}, \ldots, c_{\beta_{s}}$ are $s+1$ coefficients of $F_{0}$ linearly independent modulo $\mathfrak{m} Q$, whence $d\left(F_{0}\right) \geq s+1$, a contradiction.

Proof of Theorem 1.1. Suppose first that $N_{L}(I(0))=\mathbb{N} \times D$, as in Lemma 4.1. Then we can find $F \in \operatorname{ker} \kappa, F=\sum_{\beta \in \Delta} a_{\beta} t^{\beta}$ such that among its coefficients are $a_{\beta_{1}}, \ldots, a_{\beta_{d}}$ linearly independent modulo $\mathfrak{m} Q$. By Nakayama's Lemma, $a_{\beta_{1}}, \ldots, a_{\beta_{d}}$ generate $Q$ (recall that $d=\operatorname{dim}_{\mathbb{C}} Q / \mathfrak{m} Q$ ), so in particular they generate all the coefficients of $F$. Therefore by applying Kwieciński's Lemma 3.1 to this $F$ one obtains a nonzero $a \in \mathcal{O}_{Y}$ and a nonzero $b \in \mathcal{O}_{X\{d\}}$ with $a b=0$, i.e. a vertical component in the $d^{\prime}$ th fibre power $X^{\{d\}}$ (cf. the remark following Lemma 3.1).

Next suppose the diagram $N_{L}(I(0))$ is not trivial in any direction. Define $\widetilde{X}=$ $\mathbb{C}_{0} \times X$, and $\tilde{f}: \tilde{X} \rightarrow Y$ as $\tilde{f}=f \circ \pi$, where $\pi: \widetilde{X} \rightarrow X$ is a canonical projection. Let $\tilde{I}=I \cdot \mathcal{O}_{Y}\left\{t_{0}, t_{1}, \ldots, t_{n}\right\}$ be the extended ideal, so that $\mathcal{O}_{\widetilde{X}}=\mathcal{O}_{Y}\left\{t_{0}, t_{1}, \ldots, t_{n}\right\} / \tilde{I}$. Since every element of $\tilde{I}$ can be expressed in the form $\sum_{i \in \mathbb{N}} F_{i}\left(t_{1}, \ldots, t_{n}\right) \cdot t_{0}^{i}$, with $F_{i} \in I$, it follows that $N_{L}(\tilde{I}(0))=\mathbb{N} \times N_{L}(I(0))$. Moreover, the flattener ideal for $\tilde{f}: \tilde{X} \rightarrow Y$ is generated by the same elements as the flattener ideal $Q$, so in particular its minimal sets of generators consist of $d$ elements.

Now Lemma 4.1 applies to the diagram $N_{L}(\tilde{I}(0))$, as it is trivial in the $\beta_{0}$ direction, and following the first part of this proof we obtain that $\tilde{X}^{\{d\}}$ has a vertical component. But clearly every irreducible component $\widetilde{Z}$ of $\widetilde{X}^{\{d\}}$ is of the form $Z \times\left(\mathbb{C}^{d}\right)_{0}$ for some irreducible component $Z$ of $X^{\{d\}}$, which implies that $X^{\{d\}}$ itself has a vertical component. This completes the proof of our theorem.

\section{ACKNOWLEDGEMENTS}

I thank my Ph.D. supervisor, Professor Edward Bierstone, for endless discussions and many valuable comments that led me to the solution of the problem.

\section{REFERENCES}

[1] M. Auslander, Modules over unramified regular local rings, Illinois J.Math. 5 (1961), 631-647. MR 31:3460

[2] E.Bierstone, P.D. Milman, "The local geometry of analytic mappings", Dottorato di Ricerca in Matematica, ETS Editrice, Pisa, 1988. MR 90j:32011

[3] E.Bierstone, P. D. Milman, Flatness in analytic mappings. I. On an announcement of Hironaka, J.Geom. Anal. 1 (1991), no. 1, 19-37. MR 92c:32010

[4] N. Bourbaki, "Elements of Mathematics - Commutative Algebra", Addison - Wesley, Reading, Mass., 1972. MR 50:12997

[5] J. Frisch, Points de platitude d'un morphisme d'espaces analytiques complexes, Invent. Math. 4 (1967), 118-138. MR 36:5388

[6] A. Galligo, M. Kwieciński, Flatness and fibred powers over smooth varieties, J.Algebra 232, no.1 (2000), 48-63. MR 2001i:14006 
[7] H. Grauert, R. Remmert, "Analytische Stellenalgebren", Springer Verlag, New York, 1971. MR 47:5290

[8] H. Hironaka, Flattening theorem in complex analytic geometry, Amer. J.Math. 97 (1975), 199-265. MR 52:14365

[9] H. Hironaka, Stratification and flatness, in "Real and Complex Singularities", Proc. Oslo 1976, ed. Per Holm, Stijthof and Noordhof (1977), 199-265. MR 58:17187.

[10] H. Hironaka, M. Lejeune - Jalabert, B. Teissier, Platificateur local en géométrie analytique et aplatissement local, Astérisque 7-8 (1973), 441-446. MR 53:13636]

[11] M. Kwieciński, Flatness and fibred powers, Manuscripta Mathematica 97 (1998), 163-173. MR 99h:14017

[12] M. Kwieciński, P. Tworzewski, Fibres of analytic maps, Bull. Polish Acad. Sci. Math. 47, No. 3 (1999), 45-55. MR 2000j:14005

[13] E. Kunz, "Introduction to Commutative Algebra and Algebraic Geometry", Birkhäuser, Boston, 1985. MR 86e:14001

[14] S. Łojasiewicz, "Introduction to Complex Analytic Geometry", Birkhäuser, Basel, 1991. MR 92g:32002

[15] W. V. Vasconcelos, Flatness testing and torsionfree morphisms, Journal of Pure and Applied Algebra 122 (1997), 313-321. MR 98i:13013

[16] O. Zariski, P. Samuel, "Commutative Algebra", Van Nostrand, Princeton, 1958. MR 19:833e

Department of Mathematics, University of Toronto, Toronto, Ontario, Canada M5S 3G3

E-mail address: adamus@math.toronto.edu 